\title{
$p$-Adic Modelling of the Genome and the Genetic Code
}

\author{
Branko Dragovich $^{a *}$ and Alexandra Dragovich ${ }^{b}$ \\ ${ }^{a}$ Institute of Physics \\ P.O. Box 57, 11001 Belgrade, Serbia \\ ${ }^{b}$ Vavilov Institute of General Genetics \\ Gubkin St. 3, 119991 Moscow, Russia
}

\begin{abstract}
The present paper is devoted to foundations of $p$-adic modelling in genomics. Considering nucleotides, codons, DNA and RNA sequences, amino acids, and proteins as information systems, we have formulated the corresponding $p$-adic formalisms for their investigations. Each of these systems has its characteristic prime number used for construction of the related information space. Relevance of this approach is illustrated by some examples. In particular, it is shown that degeneration of the genetic code is a $p$-adic phenomenon. We have also put forward a hypothesis on evolution of the genetic code assuming that primitive code was based on single nucleotides and chronologically first four amino acids. This formalism of $p$-adic genomic information systems can be implemented in computer programs and applied to various concrete cases.
\end{abstract}

\section{Introduction}

Living organisms seem to be the most complex, interesting and significant objects regarding all substructures of the universe. One of the essential characteristics that differ a living organism from all other material systems is related to its genome. The genome of an organism is its whole hereditary information encoded in the desoxyribonucleic acid (DNA), and contains both genes and non-coding

\footnotetext{
* E-mail: dragovich@phy.bg.ac.yu
} 
sequences. In some viruses, which are between living and non-living objects, genetic material is encoded in the ribonucleic acid (RNA). Investigation of the entire genome is the subject of genomics. The human genome, which presents all genetic information of the Homo sapiens, is composed of more than $3 \cdot 10^{9}$ DNA base pairs and contains more than $3 \cdot 10^{4}$ genes [1]. For a more detailed and comprehensive information on molecular biology aspects of DNA, RNA and the genetic code one can use Ref. [1]. To have a self-contained exposition we shall briefly review some necessary basic properties of genomics.

The DNA is a macromolecule composed of two polynucleotide chains with a double-helical structure. Nucleotides consist of a base, a sugar and a phosphate group. Helical backbone is a result of the sugar and phosphate groups. There are four bases and they are building blocks of the genetic information. They are called adenine $(\mathrm{A})$, guanine $(\mathrm{G})$, cytosine $(\mathrm{C})$ and thymine $(\mathrm{T})$. Adenine and guanine are derived from purine, while cytosine and thymine from pyrimidine. In the sense of information, the nucleotide and its base present the same object. Nucleotides are arranged along chains of double helix through base pairs A-T and $\mathrm{C}-\mathrm{G}$ bonded by 2 and 3 hydrogen bonds, respectively. As a consequence of this pairing there is an equal number of cytosine and guanine as well as the equal rate of adenine and thymine. DNA is packaged in chromosomes which are localized in the nucleus of the eukaryotic cells.

The main role of DNA is to storage genetic information and there are two main processes to exploit this information. The first one is replication, in which DNA duplicates giving two new DNA containing the same information as the original one. This is possible owing to the fact that each of two chains contains complementary bases of the other one. The second process is related to the gene expression, i.e. the passage of DNA gene information to proteins. It performs by the messenger ribonucleic acid (mRNA), which is usually a single polynucleotide chain. The mRNA is synthesized during the first part of this process, known as transcription, when nucleotides $\mathrm{C}, \mathrm{A}, \mathrm{T}, \mathrm{G}$ from DNA are respectively transcribed into their complements $\mathrm{G}, \mathrm{U}, \mathrm{A}, \mathrm{C}$ in mRNA, where $\mathrm{T}$ is replaced by $\mathrm{U}$ ( $\mathrm{U}$ is the uracil, which is a pyrimidine). The next step in gene expression is translation, when the information coded by codons in the mRNA is translated into proteins. In this process also participate transfer tRNA and ribosomal rRNA.

Codons are ordered trinucleotides composed of C, A, U (T) and G. Each of them presents an information which controls use of one of the 20 standard amino acids or stop signal in synthesis of proteins.

Protein synthesis in all eukaryotic cells performs in the ribosomes of the cytoplasm. Proteins [2] are organic macromolecules composed of amino acids arranged in a linear chain. Amino acids are molecules that consist of amino, carboxyl and $\mathrm{R}$ (side chain) groups. Depending on $\mathrm{R}$ group there are 20 standard amino acids. 
These amino acids are joined together by a peptide bond. Proteins are substantial ingredients of all living organisms participating in various processes in cells and determining the phenotype of an organism. In the human body there may be about 2 million different proteins. The study of proteins, especially their structure and functions, is called proteomics. The proteome is the entire set of proteins in an organism.

The sequence of amino acids in a protein is determined by sequence of codons contained in DNA genes. The relation between codons and amino acids is known as the genetic code. Although there are at least 16 codes (see, e.g. [3]), the most important are two of them: the standard (eukaryotic) code and the vertebral mitochondrial code.

In the sequel we shall mainly have in mind the vertebral mitochondrial code, because it is a simple one and the others may be regarded as its slight modifications. It is obvious that there are $4 \times 4 \times 4=64$ codons. However (in the vertebral mitochondrial code), 60 of them are distributed on the 20 different amino acids and 4 make stop codons, which serve as termination signals. According to experimental observations, two amino acids are coded by six codons, six amino acids by four codons, and twelve amino acids by two codons. This property that some amino acids are coded by more than one codon is known as genetic code degeneracy. This degeneracy is a very important property of the genetic code and gives an efficient way to minimize errors caused by mutations.

Since there is in principle a huge number (between $10^{71}$ and $10^{84}$ [4]) of all possible assignments between codons and amino acids, and only a very small number of them is represented in living cells, it has been a persistent theoretical challenge to find an appropriate model explaining contemporary genetic codes. An interesting model based on the quantum algebra $\mathcal{U}_{q}(s l(2) \oplus \operatorname{sl}(2))$ in the $q \rightarrow 0$ limit was proposed as a symmetry algebra for the genetic code (see [3] and references therein). In a sense this approach mimics quark model of baryons. To describe correspondence between codons and amino-acids, it was constructed an operator which acts on the space of codons and its eigenvalues are related to amino acids. Besides some successes of this approach, there is a problem with rather many parameters in the operator. There are also papers (see, e.g. [4], [5] and [6]) starting with 64-dimensional irreducible representation of a Lie (super)algebra and trying to connect multiplicity of codons with irreducible representations of subalgebras arising in a chain of symmetry breaking. Although interesting as an attempt to describe evolution of the genetic code these Lie algebra approaches did not succeed to get its modern form. For a very brief review of these and some other theoretical approaches to the genetic code one can see Ref. [3]. There is still no generally accepted explanation of the genetic code.

It is worth recalling emergence of special theory of relativity and quantum 
mechanics. They both appeared as a result of unsatisfactory attempts to extend classical concepts to new physical phenomena, and introduction of new physical ideas and mathematical methods. Although far from everyday experience these new two theories describe physical reality quite successfully. We believe that similar situation should happen in theoretical description of living processes in biological organisms. Ultrametric and $p$-adic methods seem to be very promising tools in further investigation of the life.

Modelling of the genome, the genetic code and proteins is a challenge as well as an opportunity for applications of $p$-adic mathematical physics. Recently [7] we introduced a $p$-adic approach to DNA and RNA sequences, and to the genetic code. The central point of our approach is an appropriate identification of four nucleotides with digits 1, 2, 3, 4 of 5-adic integer expansions and application of $p$ adic distances between obtained numbers. 5-Adic numbers with three digits form 64 integers which correspond to 64 codons. In [8] we analyzed $p$-adic degeneracy of the genetic code. As one of the main results that we have obtained is explanation of the structure of the genetic code degeneracy using $p$-adic distance between

codons. A similar approach to the genetic code was considered on diadic plane [9].

Let us mention that $p$-adic models in mathematical physics have been actively considered since 1987 (see [10], [11] for early reviews and [12, [13] for some recent reviews). It is worth noting that $p$-adic models with pseudodifferential operators have been successfully applied to interbasin kinetics of proteins [14]. Some $p$-adic aspects of cognitive, psychological and social phenomena have been also considered [15]. The recent application of $p$-adic numbers in physics and related branches of sciences is reflected in the proceedings of the 2nd International Conference on $p$-Adic Mathematical Physics [16].

This paper is devoted to the further $p$-adic modelling of the genome as well as to $p$-adic roots of the genetic code evolution based on approach introduced in [7] and considered in [8].

\section{2 p-Adic Genome}

In Introduction we presented a brief review of the genome and the genetic code, as well as some motivations for their $p$-adic theoretical investigations. To consider $p$-adic properties of the genome and the genetic code in a self-contained way we shall also recall some mathematical preliminaries. 


\subsection{Some mathematical preliminaries and $p$-adic codon space}

As a new tool to study the Diophantine equations, $p$-adic numbers are introduced by German mathematician Kurt Hensel in 1897. They are involved in many branches of modern mathematics, either as rapidly developing topics or as suitable applications. An elementary introduction to $p$-adic numbers can be found in the book [17]. However, for our purposes we will use here only a small portion of $p$-adics, mainly some finite sets of integers and ultrametric distances between them.

Let us introduce the set of natural numbers

$$
\mathcal{C}_{5}[64]=\left\{n_{0}+n_{1} 5+n_{2} 5^{2}: n_{i}=1,2,3,4\right\},
$$

where $n_{i}$ are digits related to nucleotides by the following assignments: $\mathrm{C}$ (cytosine $)=1, \mathrm{~A}$ (adenine $)=2, \mathrm{~T}$ (thymine $)=\mathrm{U}$ (uracil $)=3, \mathrm{G}$ (guanine $)=4$. This is a finite expansion to the base 5 . It is obvious that 5 is a prime number and that the set $\mathcal{C}_{5}[64]$ contains 64 numbers between 31 and 124 in the usual base 10 . In the sequel we shall often denote elements of $\mathcal{C}_{5}[64]$ by their digits to the base 5 in the following way: $n_{0}+n_{1} 5+n_{2} 5^{2} \equiv n_{0} n_{1} n_{2}$. Note that here ordering of digits is the same as in the expansion, i.e this ordering is opposite to the usual one. There is now evident one-to-one correspondence between codons in three-letter notation and number $n_{0} n_{1} n_{2}$ representation.

It is also often important to know a distance between numbers. Distance can be defined by a norm. On the set $\mathbb{Z}$ of integers there are two kinds of nontrivial norm: usual absolute value $|\cdot|_{\infty}$ and $p$-adic absolute value $|\cdot|_{p}$, where $p$ is any prime number. The usual absolute value is well known from elementary mathematics and the corresponding distance between two numbers $x$ and $y$ is $d_{\infty}(x, y)=|x-y|_{\infty}$.

The $p$-adic absolute value is related to the divisibility of integers by prime numbers. Difference of two integers is again an integer. $p$-Adic distance between two integers can be understood as a measure of divisibility by $p$ of their difference (the more divisible, the shorter). By definition, $p$-adic norm of an integer $m \in \mathbb{Z}$, is $|m|_{p}=p^{-k}$, where $k \in \mathbb{N} \bigcup\{0\}$ is degree of divisibility of $m$ by prime $p$ (i.e. $\left.m=p^{k} m^{\prime}, p \nmid m^{\prime}\right)$ and $|0|_{p}=0$. This norm is a mapping from $\mathbb{Z}$ into non-negative rational numbers and has the following properties:

(i) $|x|_{p} \geq 0, \quad|x|_{p}=0$ if and only if $x=0$,

(ii) $|x y|_{p}=|x|_{p}|y|_{p}$,

(iii) $|x+y|_{p} \leq \max \left\{|x|_{p},|y|_{p}\right\} \leq|x|_{p}+|y|_{p}$ for all $x, y \in \mathbb{Z}$.

Because of the strong triangle inequality $|x+y|_{p} \leq \max \left\{|x|_{p},|y|_{p}\right\}, p$-adic absolute value belongs to non-Archimedean (ultrametric) norm. One can easily 
conclude that $0 \leq|m|_{p} \leq 1$ for any $m \in \mathbb{Z}$ and any prime $p$.

$p$-Adic distance between two integers $x$ and $y$ is

$$
d_{p}(x, y)=|x-y|_{p} .
$$

Since $p$-adic absolute value is ultrametric, the $p$-adic distance (2) is also ultrametric, i.e. it satisfies

$$
d_{p}(x, y) \leq \max \left\{d_{p}(x, z), d_{p}(z, y)\right\} \leq d_{p}(x, z)+d_{p}(z, y),
$$

where $x, y$ and $z$ are any three integers.

The above introduced set $\mathcal{C}_{5}[64]$ endowed by $p$-adic distance we shall call $p$ adic codon space, i.e. elements of $\mathcal{C}_{5}$ [64] are codons denoted by $n_{0} n_{1} n_{2}$. 5-Adic distance between two codons $a, b \in \mathcal{C}_{5}[64]$ is

$$
d_{5}(a, b)=\left|a_{0}+a_{1} 5+a_{2} 5^{2}-b_{0}-b_{1} 5-b_{2} 5^{2}\right|_{5},
$$

where $a_{i}, b_{i} \in\{1,2,3,4\}$. When $a \neq b$ then $d_{5}(a, b)$ may have three different values:

- $d_{5}(a, b)=1$ if $a_{0} \neq b_{0}$,

- $d_{5}(a, b)=1 / 5$ if $a_{0}=b_{0}$ and $a_{1} \neq b_{1}$,

- $d_{5}(a, b)=1 / 5^{2}$ if $a_{0}=b_{0}, a_{1}=b_{1}$ and $a_{2} \neq b_{2}$.

We see that the largest 5 -adic distance between codons is 1 and it is maximum $p$-adic distance on $\mathbb{Z}$. The smallest 5 -adic distance on the codon space is $5^{-2}$. Let us also note that 5-adic distance depends only on the first two nucleotides of different codons.

If we apply real (standard) distance $d_{\infty}(a, b)=\mid a_{0}+a_{1} 5+a_{2} 5^{2}-b_{0}-b_{1} 5-$ $\left.b_{2} 5^{2}\right|_{\infty}$, then third nucleotides $a_{2}$ and $b_{2}$ would play more important role than those at the second position (i.e $a_{1}$ and $b_{1}$ ), and nucleotides $a_{0}$ and $b_{0}$ are of the smallest importance. At real $\mathcal{C}_{5}[64]$ space distances are also discrete, but take values $1,2, \cdots, 93$. The smallest real and the largest 5 -adic distance are equal 1. While real distance describes metric of the ordinary physical space, this $p$-adic one serves to describe ultrametricity of the codon space.

It is worth emphasizing that the metric role of digits depends on their position in number expansion and it is quite opposite in real and $p$-adic cases. We shall see later, when we consider the genetic code, that the first two nucleotides in a codon are more important than the third one and that $p$-adic distance between codons is a natural one in description of their information content (the closer, the more similar). 


\section{$2.2 p$-Adic genomic and bioinformation spaces}

Appropriateness of the $p$-adic codon space $\mathcal{C}_{5}[64]$ to the genetic code is already shown in [8] and will be reconsidered in the Section 3. Now we want to extend $\mathcal{C}_{5}[64]$ space approach to more general genetic and bioinformation spaces.

Let us recall that four nucleotides are related to prime number 5 by their correspondence to the four nonzero digits $(1,2,3,4)$ of $p=5$. It is unappropriate to use the digit 0 for a nucleotide because it leads to non-uniqueness in representation of the codons by natural numbers. For example, $123=123000$ as numbers, but 123 represents one and 123000 two codons. This is also a reason why we do not use 4-adic representation for codons, since it would contain a nucleotide presented by digit 0 . One can use 0 as a digit to denote absence of any nucleotide.

Let us note also that we have used on $\mathcal{C}_{5}[64]$, in [7] and [8], not only 5-adic but 2-adic distance as well.

Definition 1. We shall call $(p, q)$-adic genomic space a double $\left(\Gamma_{p}[(p-\right.$ $\left.\left.1)^{m}\right], d_{q}\right)$, where

$$
\begin{array}{r}
\Gamma_{p}\left[(p-1)^{m}\right]=\left\{n_{0}+n_{1} p+\cdots+n_{m-1} p^{m-1}:\right. \\
\left.n_{i}=1,2, \cdots, p-1, m \in \mathbb{N}\right\}
\end{array}
$$

is the set of natural numbers, $d_{q}$ is the corresponding $q$-adic distance on $\Gamma_{p}[(p-$ $1)^{m}$ ] and nonzero digits $n_{i}$ are related to some $p-1$ basic constituents of a genomic system (or to any other biological information system) in a unique way. Index $q$ is a prime number.

Here $m$ can be called also multiplicity of space elements with respect to their constituents. In addition to $d_{p}$ there can be a few other $d_{q}$ useful distances on $\Gamma_{p}\left[(p-1)^{m}\right]$.

For simplicity, we shall often call $\Gamma_{p}\left[(p-1)^{m}\right] p$-adic genomic space and use notation

$$
n_{0}+n_{1} p+\cdots+n_{m-1} p^{m-1} \equiv n_{0} n_{1} \cdots n_{m-1},
$$

where ordering of digits is in the opposite direction to the standard one and seems here more natural. Earlier introduced codon space $\mathcal{C}_{5}[64]$ can be regarded as a significant example of the $p$-adic genomic spaces, i.e. $\mathcal{C}_{5}[64]=\Gamma_{5}\left[(5-1)^{3}\right]$ as space of trinucleotides. Two other examples, which will be used later, are: $\Gamma_{5}[4]$ - space of nucleotides and $\Gamma_{5}\left[4^{2}\right]$ - space of dinucleotides.

Now we can introduce $p$-adic bioinformation space as a space composed of some $p$-adic genomic spaces. 
Definition 2. Let $\mathcal{B}_{p}[N]$ be a $p$-adic space composed of $N$ natural numbers. We shall call $\mathcal{B}_{p}[N]$ p-adic bioinformation space when it can be presented as

$$
\mathcal{B}_{p}[N] \subset \prod_{m=m_{1}}^{m_{2}} \Gamma_{p}\left[(p-1)^{m}\right]
$$

where $m_{1}$ and $m_{2}$ are positive integers $\left(m_{1} \leq m_{2}\right)$, which determine the range of multiplicity between $m_{1}$ and $m_{2}$. In the sequel we shall present some concrete examples of the $\mathcal{B}_{p}[N]$ spaces.

\subsection{DNA and RNA spaces}

DNA sequences can be considered as a union of coding and non-coding segments. Coding parts are composed of codons included into genes, which are rather complex systems. In coding segments is stored information, which through a series of complex processes is translated into proteins. The space of coding DNA sequences $(c D N A)$ can be presented as

$$
c D N A[N] \subset \prod_{m=m_{1}}^{m_{2}} \Gamma_{61}\left[60^{m}\right]
$$

where $p=61$ because there are 60 codons coding amino acids (in the vertebral mitochondrial code). Thus $c D N A$ space can be regarded as a set of $N$ coded sequences as well as a set of $N$ discrete points (a lattice) of $\prod_{m=m_{1}}^{m_{2}} \Gamma_{61}\left[60^{m}\right]$ space. While in $\mathcal{C}_{5}[64]$ codons are space elements, in $c D N A[N]$ they are building units.

The structure and function of non-coding sequences is still highly unknown. They include information on various regulatory processes in the cell. We assume that the space of non-coding DNA sequences $(n c D N A)$ is a subspace

$$
n c D N A \subset \prod_{m=m_{1}}^{m_{2}} \Gamma_{5}\left[4^{m}\right]
$$

where $m_{1}$ and $m_{2}$ are minimum and maximum values of the size of non-coding segments.

In a similar way one can construct a space of all RNA sequences in the cell. 


\subsection{Protein space}

We mentioned some basic properties of proteins in Introduction. Recall also that proteins functional properties depend on their three-dimensional structure. There are four distinct levels of protein structure (primary, secondary, tertiary and quaternary) [2]. The primary structure is determined by the amino acid sequence and the other ones depend on side chains of amino acids (see Table 3). In addition to 20 standard amino acids, presented in the Table 3 , there are also 2 special nonstandard amino acids: selenocysteine and pyrrolysine [18]. They are also coded by codons, but are very rare in proteins. Thus there are 22 amino acids encoded in the genetic code. According to Jukes [19] non-freezing code may contain 28 amino acids.

The 20 standard (canonical) amino acids employed by the genetic code in proteins of the living cells are listed in Table 3. Some their important chemical properties are presented in Table 3 .

Now we want to construct an appropriate space whose elements are proteins. We propose protein space $\mathcal{P}_{p}$ to be a subspace of product of genomic spaces

$$
\mathcal{P}_{p}[N] \subset \prod_{m=m_{1}}^{m_{2}} \Gamma_{p}\left[(p-1)^{m}\right]
$$

where the building units are amino acids. Thus $\mathcal{P}_{p}[N]$ is a space of $N$ proteins with size measured by the number of amino acids between $m_{1}$ and $m_{2}\left(m_{1} \sim 10\right.$ and $m_{2} \sim 10^{4}$ ).

In (10) prime number $p$ is related to the number of amino acids by relation: $p-1=$ number of different amino acids used as building blocks in proteins. At present time there are 22 amino acids (20 standard and 2 special) and consequently $p=23$. One can argue that not all 22 amino acids have been from the very beginning of life and that has been an evolution of amino acids. Namely, using 60 different criteria for temporal order of appearance of the 20 standard amino acids the obtained result [21] is presented in Table 3. The first four amino acids (Gly, Ala, Asp and Val) have the most production rate in Miller's experiment of an imitation of the atmosphere of the early Earth. This could correspond to $p=5$ and single nucleotide codons in a primitive code. In the case of dinucleotide code there are 16 codons and maximum amino acids that can be coded is 16, i.e. $p=17$. As we already mentioned, according to Jukes [19], it is possible to code 28 amino acids by trinucleotide code and it gives the corresponding $p=29$. 


\section{3 p-Adic Genetic Code}

An intensive study of the connection between ordering of nucleotides in DNA (and RNA) and ordering of amino acids in proteins led to the experimental deciphering of the standard genetic code in the mid-1960s. The genetic code is understood as a dictionary for translation of information from DNA (through RNA) to synthesis of proteins by amino acids. The information on amino acids is contained in codons: each codon codes either an amino acid or termination signal (see, e.g. Table 3 as a standard table of the vertebral mitochondrial code). To the sequence of codons in RNA corresponds quite definite sequence of amino acids in a protein, and this sequence of amino acids determines primary structure of the protein. The genetic code is comma-free and non-overlapping. At the time of deciphering, it was mainly believed that the standard code is unique, result of a chance and fixed a long time ego. Crick [22] expressed such belief in his "frozen accident" hypothesis, which has not been supported by later observations. Moreover, it has been discovered so far at least 16 different codes and found some general regularities. At first glance the genetic code looks rather arbitrary, but it is not. Namely, mutations between synonymous codons give the same amino acid. When mutation alter an amino acid then it is like substitution of the original by similar one. In this respect the code is almost optimal.

Despite of remarkable experimental successes, there is no simple and generally accepted theoretical understanding of the genetic code. There are many papers in this direction (in addition to already cited, see also, e.g. [23] and [24]), scattered in various journals, with theoretical approaches based more or less on chemical, biological and mathematical aspects of the genetic code. Even before deciphering of the code there have been very attractive theoretical inventions (of Gamow and Crick), but the genetic code occurred to be quite different (for a review on the early inventions around the genetic code, see [25]). However, the foundation of biological coding is still an open problem. In particular, it is not clear why genetic code exists just in few known ways and not in many other possible ones. What is a principle (or principles) employed in establishment of a basic (mitochondrial) code? What are properties of codons connecting them into definite multiplets which code the same amino acid or termination signal? Answers to these and some other questions should lead us to discover an appropriate theoretical model of the genetic code. 
TABLE 1. List of 20 standard amino acids used in proteins by living cells. 3Letter and 1-letter abbreviations, and chemical structure of their side chains are presented.

\begin{tabular}{|c|c|c|}
\hline AMINO ACID & ABBR. & SIDE CHAIN (R) \\
\hline Alanine & Ala, A & $-\mathrm{CH}_{3}$ \\
\hline Cysteine & Cys, C & $-\mathrm{CH}_{2} \mathrm{SH}$ \\
\hline Aspartate & Asp, D & $-\mathrm{CH}_{2} \mathrm{COOH}$ \\
\hline Glutamate & Glu, E & $-\left(\mathrm{CH}_{2}\right)_{2} \mathrm{COOH}$ \\
\hline Phenynalanine & Phe, F & $-\mathrm{CH}_{2} \mathrm{C}_{6} \mathrm{H}_{5}$ \\
\hline Glycine & Gly, G & $-H$ \\
\hline Histidine & His, $\mathrm{H}$ & $-\mathrm{CH}_{2}-\mathrm{C}_{3} \mathrm{H}_{3} \mathrm{~N}_{2}$ \\
\hline Isoleucine & Ile, I & $-\mathrm{CH}\left(\mathrm{CH}_{3}\right) \mathrm{CH}_{2} \mathrm{CH}_{3}$ \\
\hline Lysine & Lys, K & $-\left(\mathrm{CH}_{2}\right)_{4} \mathrm{NH}_{2}$ \\
\hline Leucine & Leu, L & $-\mathrm{CH}_{2} \mathrm{CH}\left(\mathrm{CH}_{3}\right)_{2}$ \\
\hline Methionine & Met, M & $-\left(\mathrm{CH}_{2}\right)_{2} \mathrm{SCH}_{3}$ \\
\hline Asparagine & Asn, $\mathrm{N}$ & $-\mathrm{CH}_{2} \mathrm{CONH}_{2}$ \\
\hline Proline & Pro, $\mathrm{P}$ & $-\left(\mathrm{CH}_{2}\right)_{3^{-}}$ \\
\hline Glutamine & Gln, Q & $-\left(\mathrm{CH}_{2}\right)_{2} \mathrm{CONH}_{2}$ \\
\hline Arginine & Arg, R & $-\left(\mathrm{CH}_{2}\right)_{3} \mathrm{NHC}(\mathrm{NH}) \mathrm{NH}_{2}$ \\
\hline Serine & Ser, S & $-\mathrm{CH}_{2} \mathrm{OH}$ \\
\hline Threonine & Thr, T & $-\mathrm{CH}(\mathrm{OH}) \mathrm{CH}_{3}$ \\
\hline Valine & Val, V & $-\mathrm{CH}\left(\mathrm{CH}_{3}\right)_{2}$ \\
\hline Tryptophan & Trp, W & $-\mathrm{CH}_{2} \mathrm{C}_{8} \mathrm{H}_{6} \mathrm{~N}$ \\
\hline Tyrosine & Tyr, Y & $-\mathrm{CH}_{2}-\mathrm{C}_{6} \mathrm{H}_{4} \mathrm{OH}$ \\
\hline
\end{tabular}


TABLE 2. Some chemical properties of 20 standard amino acids. $p+n$ is number of nucleons. These and some other recent chemical values can be found in [20].

\begin{tabular}{|l|r|l|l|l|}
\hline $\begin{array}{l}\text { Amino } \\
\text { acids }\end{array}$ & p+n & Polar & $\begin{array}{l}\text { Hydro- } \\
\text { phobic }\end{array}$ & $\begin{array}{c}\text { In proteins } \\
\%\end{array}$ \\
\hline Ala, A & 89 & no & yes & 7.8 \\
Cys, C & 121 & no & yes & 1.9 \\
Asp, D & 133 & yes & no & 5.3 \\
Glu, E & 147 & yes & no & 6.3 \\
Phe, F & 165 & no & yes & 3.9 \\
Gly, G & 75 & no & yes & 7.2 \\
His, H & 155 & yes & no & 2.3 \\
Ile, I & 131 & no & yes & 5.3 \\
Lys, K & 146 & yes & no & 5.9 \\
Leu, L & 131 & no & yes & 9.1 \\
Met, M & 149 & no & yes & 2.3 \\
Asn, N & 132 & yes & no & 4.3 \\
Pro, P & 115 & no & yes & 5.2 \\
Gln, Q & 146 & yes & no & 4.2 \\
Arg, R & 174 & yes & no & 5.1 \\
Ser, S & 105 & yes & no & 6.8 \\
Thr, T & 119 & yes & no & 5.9 \\
Val, V & 117 & no & yes & 6.6 \\
Trp, W & 204 & no & yes & 1.4 \\
Tyr, Y & 181 & yes & yes & 3.2 \\
\hline
\end{tabular}

TABLE 3. Temporal appearance of the 20 standard amino acids [21].

\begin{tabular}{llll}
\hline (1) Gly & (2) Ala & (3) Asp & (4) Val \\
(5) Pro & (6) Ser & (7) Glu & (8) Leu \\
(9) Thr & (10) Arg & (11) Ile & (12) Gln \\
(13) Asn & (14) His & (15) Lys & (16) Cys \\
(17) Phe & (18) Tyr & (19) Met & (20) Trp \\
\hline
\end{tabular}


TABLE 4. The standard (Watson-Crick) table of the vertebral mitochondrial code. Ter denotes the terminal (stop) signal.

\begin{tabular}{|ll|ll|ll|ll|}
\hline UUU & Phe & UCU & Ser & UAU & Tyr & UGU & Cys \\
UUC & Phe & UCC & Ser & UAC & Tyr & UGC & Cys \\
UUA & Leu & UCA & Ser & UAA & Ter & UGA & Trp \\
UUG & Leu & UCG & Ser & UAG & Ter & UGG & Trp \\
\hline CUU & Leu & CCU & Pro & CAU & His & CGU & Arg \\
CUC & Leu & CCC & Pro & CAC & His & CGC & Arg \\
CUA & Leu & CCA & Pro & CAA & Gln & CGA & Arg \\
CUG & Leu & CCG & Pro & CAG & Gln & CGG & Arg \\
\hline AUU & Ile & ACU & Thr & AAU & Asn & AGU & Ser \\
AUC & Ile & ACC & Thr & AAC & Asn & AGC & Ser \\
AUA & Met & ACA & Thr & AAA & Lys & AGA & Ter \\
AUG & Met & ACG & Thr & AAG & Lys & AGG & Ter \\
\hline GUU & Val & GCU & Ala & GAU & Asp & GGU & Gly \\
GUC & Val & GCC & Ala & GAC & Asp & GGC & Gly \\
GUA & Val & GCA & Ala & GAA & Glu & GGA & Gly \\
GUG & Val & GCG & Ala & GAG & Glu & GGG & Gly \\
\hline
\end{tabular}


TABLE 5. Our table of the vertebral mitochondrial code in the usual notation.

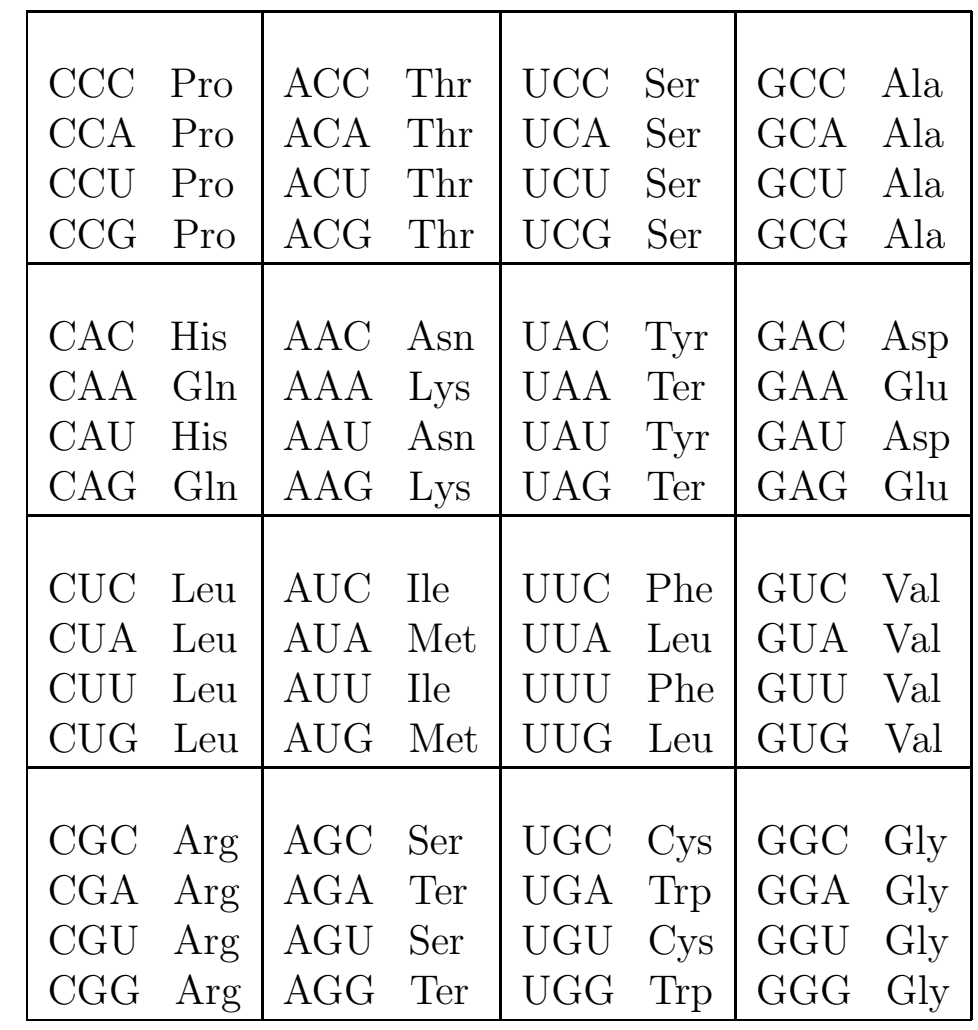


TABLE 6. Our 5-adic table of the vertebral mitochondrial code, which is a representation of the $\mathcal{C}_{5}[64]$ codon space.

\begin{tabular}{|ll|ll|ll|ll|}
\hline 111 & Pro & 211 & Thr & 311 & Ser & 411 & Ala \\
112 & Pro & 212 & Thr & 312 & Ser & 412 & Ala \\
113 & Pro & 213 & Thr & 313 & Ser & 413 & Ala \\
114 & Pro & 214 & Thr & 314 & Ser & 414 & Ala \\
\hline & & & & & & & \\
121 & His & 221 & Asn & 321 & Tyr & 421 & Asp \\
122 & Gln & 222 & Lys & 322 & Ter & 422 & Glu \\
123 & His & 223 & Asn & 323 & Tyr & 423 & Asp \\
124 & Gln & 224 & Lys & 324 & Ter & 424 & Glu \\
\hline & & & & & & & \\
131 & Leu & 231 & Ile & 331 & Phe & 431 & Val \\
132 & Leu & 232 & Met & 332 & Leu & 432 & Val \\
133 & Leu & 233 & Ile & 333 & Phe & 433 & Val \\
134 & Leu & 234 & Met & 334 & Leu & 434 & Val \\
\hline & & & & & & & \\
141 & Arg & 241 & Ser & 341 & Cys & 441 & Gly \\
142 & Arg & 242 & Ter & 342 & Trp & 442 & Gly \\
143 & Arg & 243 & Ser & 343 & Cys & 443 & Gly \\
144 & Arg & 244 & Ter & 344 & Trp & 444 & Gly \\
\hline
\end{tabular}


Let us now turn to Table 3. We observe that this table can be regarded as a big rectangle divided into 16 equal smaller rectangles: 8 of them are quadruplets which one-to-one correspond to 8 amino acids, and other 8 rectangles are divided into 16 doublets coding 14 amino acids and termination (stop) signal (by two doublets at different places). However there is no manifest symmetry in distribution of these quadruplets and doublets.

In order to get a symmetry we have rewritten this standard table into new one by rearranging 16 rectangles. As a result we obtained Table 3 which exhibits a symmetry with respect to the distribution of codon quadruplets and codon doublets. Namely, in our table quadruplets and doublets form separately two figures, which are symmetric with respect to the mid vertical line (a left-right symmetry), i.e. they are invariant under interchange $C \leftrightarrow G$ and $A \leftrightarrow U$ at the first position in codons at all horizontal lines. Recall that also DNA is symmetric under simultaneous interchange of complementary nucleotides $C \leftrightarrow G$ and $A \leftrightarrow T$ between its strands. All doublets in this table form a nice figure which looks like letter $\mathbb{T}$.

Table 3 contains the same distribution of amino acids as Table 3 , but codons are now presented by 5-adic numbers $n_{0} n_{1} n_{2}$ instead of capital letters (recall: $\mathrm{C}$ $=1, \mathrm{~A}=2, \mathrm{U}=3, \mathrm{G}=4)$. This new table can be also regarded as a representation of the $\mathcal{C}_{5}$ [64] codon space with gradual increasing of integers from 111 to 444. The observed left-right symmetry is now invariance under the corresponding transformations $1 \leftrightarrow 4$ and $2 \leftrightarrow 3$. In other words, at each horizontal line one can perform doublet $\leftrightarrow$ doublet and quadruplet $\leftrightarrow$ quadruplet interchange around vertical midline.

It is worth noting that the above invariance leaves also unchanged polarity and hydrophobicity of the corresponding amino acids in all but three cases: Asn $\leftrightarrow$ Tyr, Arg $\leftrightarrow$ Gly, and Ser $\leftrightarrow$ Cys.

\subsection{Degeneracy of the genetic code}

Let us now explore distances between codons and their role in formation of the genetic code degeneration.

To this end let us again turn to Table 3 as a representation of the $\mathcal{C}_{5}$ [64] codon space. Namely, we observe that there are 16 quadruplets such that each of them has the same first two digits. Hence 5-adic distance between any two different codons within a quadruplet is

$$
\begin{gathered}
d_{5}(a, b)=\left|a_{0}+a_{1} 5+a_{2} 5^{2}-a_{0}-a_{1} 5-b_{2} 5^{2}\right|_{5} \\
=\left|\left(a_{2}-b_{2}\right) 5^{2}\right|_{5}=\left|\left(a_{2}-b_{2}\right)\right|_{5}\left|5^{2}\right|_{5}=5^{-2}
\end{gathered}
$$


because $a_{0}=b_{0}, a_{1}=b_{1}$ and $\left|a_{2}-b_{2}\right|_{5}=1$. According to (11) nucleotides within every quadruplet are at the smallest distance, i.e. they are closest comparing to all other nucleotides.

Since codons are composed of three arranged nucleotides, each of which is either a purine or a pyrimidine, it is natural to try to quantify similarity inside purines and pyrimidines, as well as distinction between elements from these two groups of nucleotides. Fortunately there is a tool, which is again related to the $p$-adics, and now it is 2 -adic distance. One can easily see that 2 -adic distance between pyrimidines $\mathrm{C}$ and $\mathrm{U}$ is $d_{2}(1,3)=|3-1|_{2}=1 / 2$ as the distance between purines $\mathrm{A}$ and $\mathrm{G}$, namely $d_{2}(2,4)=|4-2|_{2}=1 / 2$. However 2-adic distance between $\mathrm{C}$ and $\mathrm{A}$ or $\mathrm{G}$ as well as distance between $\mathrm{U}$ and $\mathrm{A}$ or $\mathrm{G}$ is 1 (i.e. maximum).

With respect to 2-adic distance, the above quadruplets may be regarded as composed of two doublets: $a=a_{0} a_{1} 1$ and $b=a_{0} a_{1} 3$ make the first doublet, and $c=a_{0} a_{1} 2$ and $d=a_{0} a_{1} 4$ form the second one. 2-Adic distance between codons within each of these doublets is $\frac{1}{2}$, i.e.

$$
d_{2}(a, b)=\left|(3-1) 5^{2}\right|_{2}=\frac{1}{2}, \quad d_{2}(c, d)=\left|(4-2) 5^{2}\right|_{2}=\frac{1}{2},
$$

because $3-1=4-2=2$.

One can now look at Table 3 as a system of 32 doublets. Thus 64 codons are clustered by a very regular way into 32 doublets. Each of 21 subjects (20 amino acids and 1 termination signal) is coded by one, two or three doublets. In fact, there are two, six and twelve amino acids coded by three, two and one doublet, respectively. Residual two doublets code termination signal.

Note that 2 of 16 doublets code 2 amino acids (Ser and Leu) which are already coded by 2 quadruplets, thus amino acids Serine and Leucine are coded by 6 codons (3 doublets).

To have a more complete picture on the genetic code it is useful to consider possible distances between codons of different quadruplets as well as between different doublets. Also, we introduce distance between quadruplets or between doublets, especially when distances between their codons have the same value. Thus 5-adic distance between any two quadruplets in the same column is $1 / 5$, while such distance between other quadruplets is 1. 5-Adic distance between doublets coincides with distance between quadruplets, and this distance is $\frac{1}{5^{2}}$ when doublets are within the same quadruplet.

The 2-adic distances between codons, doublets and quadruplets are more complex. There are three basic cases:

- codons differ only in one digit, 
- codons differ in two digits,

- codons differ in all three digits.

In the first case, 2-adic distance can be $\frac{1}{2}$ or 1 depending whether difference between digits is 2 or not, respectively.

Let us now look at 2-adic distances between doublets coding leucine and also between doublets coding serine. These are two cases of amino acids coded by three doublets. One has the following distances:

- $d_{2}(332,334)=d_{2}(132,134)=\frac{1}{2}$ for leucine,

- $d_{2}(311,241)=d_{2}(313,243)=\frac{1}{2}$ for serine.

If we use usual distance between codons, instead of $p$-adic one, then we would observe that two synonymous codons are very far (at least 25 units), and that those which are close code different amino acids. Thus we conclude that not usual metric but ultrametric is inherent to codons.

How degeneracy of the genetic code is connected with $p$-adic distances between codons? The answer is in the following $p$-adic degeneracy principle: Two codons have the same meaning with respect to amino acids if they are at smallest 5-adic and 0.5 2-adic distance. Here $p$-adic distance plays a role of similarity: the closer, the more similar. Taking into account all known codes (see the next subsection) there is a slight violation of this principle. Now it is worth noting that in modern particle physics just broken of the fundamental gauge symmetry gives its standard model. There is a sense to introduce a new principle (let us call it reality principle): Reality is realization of some broken fundamental principles. It seems that this principle is valid not only in physics but also in all sciences. In this context modern genetic code is an evolutionary broken the above $p$-adic degeneracy principle.

\subsection{Evolution of the genetic code}

The origin and early evolution of the genetic code are among the most interesting and important investigations related to the origin and whole evolution of the life. However, since there are no concrete facts from that early period, it gives rise to many speculations. Nevertheless, one can hope that some of the hypotheses may be tested looking for their traces in the contemporary genomes.

It seems natural to consider biological evolution as an adaptive development of simpler living systems to more complex ones. Namely, living organisms are open systems in permanent interaction with environment. Thus the evolution 
can be modelled by a system with given initial conditions and guided by some internal rules taking into account environmental factors.

We are going now to conjecture on the evolution of the genetic code using our p-adic approach to the genomic space, and assuming that preceding codes used simpler codons and older amino acids.

Recall that $p$-adic genomic space $\Gamma_{p}\left[(p-1)^{m}\right]$ has two parameters: $p$-related to $p-1$ building blocks, and $m$ - multiplicity of the building blocks in space elements.

- Case $\Gamma_{2}[1]$ is a trivial one and useless for a primitive code.

- Case $\Gamma_{3}\left[2^{m}\right]$ with $m=1,2,3$ does not seem to be realistic.

- Case $\Gamma_{5}\left[4^{m}\right]$ with $m=1,2,3$ offers a possible pattern to consider evolution of the genetic code. Namely, the codon space could evolve in the following way: $\Gamma_{5}[4] \rightarrow \Gamma_{5}\left[4^{2}\right] \rightarrow \Gamma_{5}\left[4^{3}\right]=\mathcal{C}_{5}[64]$ (see also Table 3.2 ).

According to Table 3 this primary code, containing codons in the single nucleotide form (C, A, U, G), encoded the first four amino acids: Gly, Ala, Asp and Val. From the last column of Table 3 we conclude that the connection between digits and amino acids is: $1=$ Ala, $2=$ Asp, $3=$ Val, $4=$ Gly. In the primary code these digits occupied the first position in the 5-adic expansion (Table 3.2), and at the next step, i.e. $\Gamma_{5}[4] \rightarrow \Gamma_{5}\left[4^{2}\right]$, they moved to the second position adding digits 1,2,3,4 in front of each of them.

In $\Gamma_{5}\left[4^{2}\right]$ one has 16 dinucleotide codons which can code up to 16 new amino acids. Addition of the digit 4 in front of already existing codons 1,2,3, 4 leaves their meaning unchanged, i.e. $41=$ Ala, $42=$ Asp, $43=$ Val, and $44=$ Gly. Adding digits 3, 2, 1 in front of the primary 1,2,3, 4 codons one obtains 12 possibilities for coding some new amino acids. To decide which amino acid was encoded by which of 12 dinucleotide codons, we use as a criterion their immutability in the trinucleotide coding on the $\Gamma_{5}\left[4^{3}\right]$ space. This criterion assumes that amino acids encoded earlier are more fixed than those encoded later. According to this criterion we decide in favor of the first row in each rectangle of Table 3 and result is presented in Table 3.2 .

Transition from dinucleotide to trinucleotide codons occurred by attaching nucleotides 1, 2, 3, 4 at the third position, i. e. behind each dinucleotide. By this way one obtains new codon space $\Gamma_{5}\left[4^{3}\right]=\mathcal{C}_{5}[64]$, which is significantly enlarged and provides a pattern to generate known genetic codes. This codon space $\mathcal{C}_{5}[64]$ gives possibility to realize at least three general properties of the modern code:

(i) encoding of more than 16 amino acids,

(ii) diversity of codes, 
(iii) stability of the gene expression.

Let us give some relevant clarifications.

(i) For functioning of contemporary living organisms it is necessary to code at least 20 standard (Table 3) and 2 non-standard amino acids (selenocysteine and pyrrolysine). Probably these 22 amino acids are also sufficient building units for biosynthesis of all necessary contemporary proteins. While $\Gamma_{5}\left[4^{2}\right]$ is insufficient, the genomic space $\Gamma_{5}\left[4^{3}\right]$ offers approximately three codons per one amino acid.

(ii) The eukariotic (often called standard or universal) code is established around 1966 and was thought to be universal, i. e., common to all organisms. When the vertebral mitochondrial code was discovered in 1979, it gave rise to believe that the code is not frozen and that there are also some other codes which are mutually different. According to later evidences, one can say that there are at least 16 slightly different mitochondrial and nuclear codes (for a review, see [26], [27] and references therein). Different codes have some codons with different meaning. So, in the standard code there are the following changes in Table 3 :

- 232 (AUA): Met $\rightarrow$ Ile,

- $242(\mathrm{AGA})$ and $244(\mathrm{AGG}):$ Ter $\rightarrow$ Arg,

- 342 (UGA): Trp $\rightarrow$ Ter.

Modifications in this 16 codes are not homogeneously distributed on 16 rectangles of Table 3. For instance, in all 16 codes codons $41 i(i=1,2,3,4)$ have the same meaning.

(iii) Each of the 16 codes is degenerate and degeneration provides their stability against possible mutations. In other words, degeneration helps to minimize codon errors.

Genetic codes based on single nucleotide and dinucleotide codons were mainly directed to code amino acids with rather different properties. This may be the reason why amino acids Glu and Gln are not coded in dinucleotide code (Table 3.2), since they are similar to Asp and Asn, respectively. However, to become almost optimal, trinucleotide codes have taken into account structural and functional similarities of amino acids.

We presented here a hypothesis on the genetic code evolution taking into account possible codon evolution, from 1-nucleotide to 3-nucleotide, and amino acids temporal appearance. This scenario may be extended to the cell evolution, which probably should be considered as a coevolution of all its main ingredients (for an early idea of the coevolution, see [28]). 
TABLE 7. 5-Adic system including digit 0, and containing single nucleotide, dinucleotide and trinucleotide codons. If one ignores numbers which contain digit 0 in front of any 1, 2, 3 or 4, then one has one-to-one correspondence between 1-digit, 2-digits, 3-digits numbers and single nucleotides, dinucleotides, trinucleotides, respectively. It seems that evolution of codons has followed transitions: single nucleotides $\rightarrow$ dinucleotides $\rightarrow$ trinucleotides.

\begin{tabular}{|c|c|c|c|c|}
\hline 000 & $100 \mathrm{C}$ & $200 \mathrm{~A}$ & $300 \mathbf{U}$ & $400 \mathrm{G}$ \\
\hline 010 & $110 \mathrm{CC}$ & $210 \mathrm{AC}$ & $310 \mathbf{U C}$ & $410 \mathbf{G C}$ \\
\hline 020 & $120 \mathbf{C A}$ & $220 \mathbf{A A}$ & $320 \mathbf{U A}$ & $420 \mathbf{G A}$ \\
\hline 030 & $130 \mathrm{CU}$ & $230 \mathrm{AU}$ & $330 \mathbf{U U}$ & $430 \mathbf{G U}$ \\
\hline 040 & $140 \mathrm{CG}$ & $240 \mathrm{AG}$ & $340 \mathbf{U G}$ & $440 \mathbf{G G}$ \\
\hline 001 & 101 & 201 & 301 & 401 \\
\hline 011 & $111 \mathrm{CCC}$ & $211 \mathrm{ACC}$ & 311 UCC & 411 GCC \\
\hline 021 & $121 \mathrm{CAC}$ & $221 \mathbf{A A C}$ & 321 UAC & $421 \mathbf{G A C}$ \\
\hline 031 & $131 \mathbf{C U C}$ & 231 AUC & 331 UUC & 431 GUC \\
\hline 041 & $141 \mathrm{CGC}$ & 241 AGC & 341 UGC & 441 GGC \\
\hline 002 & 102 & 202 & 302 & 402 \\
\hline 012 & 112 CCA & 212 ACA & 312 UCA & 412 GCA \\
\hline 022 & 122 CAA & 222 AAA & 322 UAA & 422 GAA \\
\hline 032 & 132 CUA & 232 AUA & 332 UUA & 432 GUA \\
\hline 042 & 142 CGA & 242 AGA & 342 UGA & 442 GGA \\
\hline 003 & 103 & 203 & 303 & 403 \\
\hline 013 & $113 \mathrm{CCU}$ & 213 ACU & $313 \mathbf{U C U}$ & 413 GCU \\
\hline 023 & $123 \mathrm{CAU}$ & $223 \mathbf{A A U}$ & 323 UAU & 423 GAU \\
\hline 033 & 133 CUU & 233 AUU & $333 \mathbf{U U U}$ & 433 GUU \\
\hline 043 & 143 CGU & 243 AGU & 343 UGU & 443 GGU \\
\hline 004 & 104 & 204 & 304 & 404 \\
\hline 014 & $114 \mathrm{CCG}$ & 214 ACG & 314 UCG & 414 GCG \\
\hline 024 & $124 \mathbf{C A G}$ & 224 AAG & $324 \mathbf{U A G}$ & 424 GAG \\
\hline 034 & $134 \mathbf{C U G}$ & 234 AUG & $334 \mathbf{U U G}$ & 434 GUG \\
\hline 044 & 144 CGG & 244 AGG & 344 UGG & 444 GGG \\
\hline
\end{tabular}

21 
TABLE 8. The dinucleotide genetic code based on the p-adic genomic space $\Gamma_{5}\left[4^{2}\right]$. Note that it encodes 15 amino acids without stop codon, but encoding serine twice.

\begin{tabular}{|ll|ll|ll|ll|}
\hline 11 & Pro & 21 & Thr & 31 & Ser & 41 & Ala \\
\hline 12 & His & 22 & Asn & 32 & Tyr & 42 & Asp \\
\hline 13 & Leu & 23 & Ile & 33 & Phe & 43 & Val \\
\hline & & & & & & & \\
14 & Arg & 24 & Ser & 34 & Cys & 44 & Gly \\
\hline
\end{tabular}

\section{Concluding Remarks}

There are two aspects of the genetic code related to:

(i) multiplicity of codons which code the same amino acid,

(ii) concrete assignment of codon multiplets to particular amino acids.

The above presented $p$-adic approach gives quite satisfactory description of the aspect (i). Ultrametric behavior of $p$-adic distances between elements of the $\mathcal{C}_{5}[64]$ codon space radically differs from the usual ones. Quadruplets and doublets of codons have natural explanation within 5-adic and 2-adic nearness. Degeneracy of the genetic code in the form of doublets, quadruplets and sextuplets is direct consequence of $p$-adic ultrametricity between codons. $p$-Adic $\mathcal{C}_{5}$ [64] codon space is our theoretical pattern to consider all variants of the genetic code: some codes are direct representation of $\mathcal{C}_{5}[64]$ and the others are its slight evolutional modifications.

(ii) Which amino acid corresponds to which multiplet of codons? An answer to this question should be expected from connections between physicochemical properties of amino acids and anticodons. Namely, enzyme aminoacyl-tRNA synthetase links specific tRNA anticodon and related amino acid. Thus there is no direct interaction between amino acids and codons, as it was believed for some time in the past.

Note that there are in general 4 ! ways to assign digits $1,2,3,4$ to nucleotides $\mathrm{C}, \mathrm{A}, \mathrm{U}, \mathrm{G}$. After an analysis of all 24 possibilities, we have taken $\mathrm{C}=1, \mathrm{~A}=2$, $\mathrm{U}=\mathrm{T}=3, \mathrm{G}=4$ as a quite appropriate choice. In addition to various properties already presented in this paper it exhibits also complementarity of nucleotides in the DNA double helix by relation $\mathrm{C}+\mathrm{G}=\mathrm{A}+\mathrm{T}=5$.

It would be useful to find an analogous connection between 22 amino acids 
and digits $1,2, \cdots, 22$ in $p=23$ representation. Now there are 22 ! possibilities and to explore all of them seems to be a hard task. However, use of computer analysis may help to find a satisfactory solution.

One can express many above considerations of $p$-adic information theory in linguistic terms and investigate possible linguistic applications.

In this paper we have employed $p$-adic distances to measure similarity between codons, which have been used to describe degeneracy of the genetic code. It is worth noting that in other contexts $p$-adic distances can be interpreted in quite different meanings. For example, 3 -adic distance between cytosine and guanine is $d_{3}(1,4)=\frac{1}{3}$, and between adenine and thymine $d_{3}(2,3)=1$. This 3 -adic distance seems to be natural to relate to hydrogen bonds between complements in DNA double helix: the smaller distance, the stronger hydrogen bond. Recall that $\mathrm{C}-\mathrm{G}$ and $\mathrm{A}-\mathrm{T}$ are bonded by 3 and 2 hydrogen bonds, respectively.

The translation of codon sequences into proteins is highly an informationprocessing phenomenon. $p$-Adic information modelling presented in this paper offers a new approach to systematic investigation of ultrametric aspects of DNA and RNA sequences, the genetic code and the world of proteins. It can be embedded in computer programs to explore $p$-adic side of the genome and related subjects.

The above considerations and obtained results may be regarded as contributions mainly towards foundations of (i) p-adic theory of information and (ii) $p$-adic theory of the genetic code.

Summarizing, contributions to

(i) $p$-adic theory of information contain:

- formulation of $p$-adic genomic space (whose examples are spaces of nucleotides, dinucleotides and trinucleotides),

- formulation of $p$-adic bioinformation space (whose examples are DNA, RNA and protein spaces),

- relation between building blocks of information spaces and some prime numbers;

(ii) $p$-adic theory of the genetic code include:

- description of codon quadruplets and doublets by 5-adic and 2-adic distances,

- observation of a symmetry between quadruplets as well as between doublets at our table of codons,

- formulation of degeneracy principle, 
- formulation of hypothesis on codon evolution.

Many problems remain to be explored in the future on the above $p$-adic approach to genomics. Among the most attractive and important themes are:

- elaboration of the $p$-adic theory of information towards genomics,

- evolution of the genome and the genetic code,

- structure and function of non-coding DNA,

- ultrametric aspects of proteins,

- creation of the corresponding computer programs.

\section{Acknowledgement}

The work on this paper was partially supported by the Ministry of Science, Serbia, contract 144032D, the Russian Foundation for Basic Research, grant RFFI 06-04-08244, and Program of the Russian Academy of Sciences "Dynamics of plants, animals and human genofond". B.D. would like to thank M. Rakocevic for discussions on chemical aspects of the genetic code.

\section{References}

[1] Watson, J.D., Baker, T.A., Bell, S.P., Gann, A., Levine M. and Losick, R. (2004) Molecular Biology of the Gene. CSHL Press, Benjamin Cummings, San Francisco.

[2] Finkelshtein, A.V. and Ptitsyn, O.B. (2002) Physics of Proteins. Academic Press, London.

[3] Frappat, L., Sciarrino, A. and Sorba, P. (2001) Crystalizing the Genetic Code. J. Biol. Phys., 27, 1-38. arXiv:physics/0003037.

[4] Hornos, J.E.M. and Hornos, Y.M.M. (1993) Algebraic Model for the Evolution of the Genetic Code. Phys. Rev. Lett., 71, 4401-4404.

[5] Forger, M. and Sachse, S. (2000) Lie Superalgebras and the Multiplet Structure of the Genetic Code I: Codon Representations. J. Math. Phys., 41 (8), 5407 - 5422. arXiv:math-ph/9808001. 
[6] Bashford, J.D., Tsohantjis, I., Jarvis, P.D. (1997) Codon and nucleotide assignments in a supersymmetric model of the genetic code. Phys. Lett. A, 233, $481-488$.

[7] Dragovich, B. and Dragovich, A. (2006) A p-Adic Model of DNA Sequence and Genetic Code. arXiv:q-bio.GN/0607018v1.

[8] Dragovich, B. and Dragovich, A. (2007) p-Adic Degeneracy of the Genetic Code. SFIN A, 20 (1), 179 - 188. arXiv:0707.0764v1 [q-bio.GN].

[9] Khrennikov, A. and Kozyrev, S. (2007) Genetic code on a diadic plane. arXiv:q-bio/0701007.

[10] Brekke, L. and Freund, P.G.O. (1993) p-adic numbers in physics. Phys. Rept., 233, 1-66.

[11] Vladimirov, V.S., Volovich I.V. and Zelenov, E.I. (1994) p-Adic Analysis and Mathematical Physics. World Scientific, Singapore.

[12] Dragovich, B. (2004) p-Adic and Adelic Quantum Mechanics. Proc. V. A. Steklov Inst. Math. , 245, 72 - 85. arXiv:hep-th/0312046.

[13] Dragovich, B. (2006) p-Adic and Adelic Cosmology: p-Adic Origin of Dark Energy and Dark Matter. p-Adic Mathematical Physics. AIP Conference Proceedings, 826, 25 - 42. arXiv:hep-th/0602044.

[14] Avetisov, V.A., Bikulov, A.Kh., Kozyrev, S.V. and Osipov, V.A. (2002) p-Adic Models of Ultrametric Diffusion Constrained by Hierarchical Energy Landscape. J. Phys. A: Math. Gen., 35 (2), 177 - 189. arXiv:cond-mat/0106506.

[15] Khrennikov, A. (2004) Information Dynamics in Cognitive, Psychological, Social and Anomalous Phenomena. Kluwer AP, Dordrecht.

[16] (2006) p-Adic Mathematical Physics, Proceedings of the 2nd International Conference on $p$-Adic Mathematical Physics. AIP Conference Proceedings, 826 .

[17] Gouvea, F.Q. (1993) p-Adic numbers: An introduction. (Universitext), Springer, Berlin.

[18] Wikipedia (2007) Amino acid. http://en.wikipedia.org/wiki/Amino_acid.

[19] Jukes, T.H. (1983) Evolution of the amino acid code: inferences from mitochondrial codes. J. Mol. Evol., 19, 219 - 225. 
[20] Wikipedia (2007) List of standard amino acids. http://en.wikipedia.org/wiki/List_of_standard_amino_acids.

[21] Trifonov, E.N. (2004) The Triplet Code From First Principles. Journal of Biomolecular Structure and Dynamics, 22 (1), 1-11.

[22] Crick, F. (1968) The Origin of the Genetic Code. J. Mol. Biol, 38, $367-$ 379.

[23] Rumer, Yu.B. (1966) On Systematization of Codons in the Genetic Code. Doklady Acad. Nauk USSR, 167 (6), 1393 - 1394.

[24] Swanson, R. (1984) A Unifying Concept for the Amino Acid Code. Bull. Math. Biol., 46 (2), $187-203$.

[25] Hayes, B. (1998) The Invention of the Genetic Code. American Scientist, 86 (1), $8-14$.

[26] Knight, R.D., Freeland, S.J. and Landweber, L.F. (2001) Rewiring the Keyboard: Evolvability of the Genetic Code. Nat. Rev. Genet., 2, 49 - 58.

[27] Osawa, S., Jukes, T.H., Watanabe, K., and Muto, A. (1992) Recent Evidence for Evolution of the Genetic Code. Microb. Rev., 56 (1), 229 - 264.

[28] Wong, J.T.F. (1975) A Co-Evolution Theory of the Genetic Code. Proc. Nat. Acad. Sci. USA, 72, $1909-1912$. 